\title{
Performance Analysis of Error Control Codes for Underwater Wireless Acoustic Communication
}

\author{
Salma S. Shahapur ${ }^{1, *}$, Rajashri Khanai ${ }^{2}$ and Dattaprasad A. Torse ${ }^{3}$ \\ ${ }^{I}$ Department of Electronics and Communication, Jain College of Engineering, Belagavi, Karnataka, India \\ ${ }^{2}$ Department of Electronics and Communication, KLE DR. MSSCET College of Engineering and Technology, \\ Karnataka, India \\ ${ }^{3}$ Department of Electronics and Communication, Gogte Institute of Technology Belagavi, Karnataka, India
}

('Corresponding author's e-mail: salma.jce@gmail.com)

Received: 18 September 2020, Revised: 22 May 2021, Accepted: 18 June 2021

\begin{abstract}
In underwater acoustic communication, the information transmitted from 1 sensor node to another is corrupted due to errors persuaded by the noisy channel and other issues. To reduce the bit error rate, it is essential to propose suitable error regulator structure. In this paper, we simulate the performance analysis of Orthogonal Frequency Division Multiplexing Interleaver Division Multiple Access Multiple Input Multiple Output scheme with different channel codes to improve bit error rate performance. Bit error rate and consumed power are measured by communicating arbitrarily generated information through AWGN network. From the simulation results and assessment of the 2 divergent channel coding, 2 interleavers and 3 modulation techniques. We conclude that turbo codes with random interleaver and binary phase shift keying are best suitable to improve reliability performance for underwater wireless acoustic communication. To reduce the burst error in underwater acostic communication we propose an hybrid approach IDMA OFDM MIMO. BER performance is improved upto $10^{-6}$.
\end{abstract}

Keywords: Convolutional coding, Matrix interleaving, OFDM MIMO, Turbo coding

\section{Introduction}

The information send through the acoustic channel is exposed to manipulation due to noise, to decrease the bit error rate, error control systems are required. In underwater acoustic communication because of severe energy limitation, we cannot rise transmitted power of the signal. Hence to reduce bit error rate another method is we can use the error control codes. Encoder circuit, decoder circuit of error controller codes demolish ample quantity of energy. This encourages us to learn error finding and error improvement codes. The 2 main treats in communication system are Additive White Gaussian Noise (AWGN) and interference. Years of analysis, we will handle Additive White Gaussian Noise mistreatment committal to inscription techniques (forward error improvement codes) like low-density redundant check codes [1] and turbo codes [2]. Interference continues to be a problematic, the advance is being created progressively [3-5]. In communication system Interference could are available completely in different forms, example;

1) Interference because of multiple-access,

2) In multipath channels lay to reference because of inter image interference,

3) In multiple transmit antenna systems interference because of cross antenna interference.

We will think about interference as additive noise; this principle is taken for capricious code division multiple access wave form schemes in single-user detection [6]. In scientific theory, for communication apply of random cryptography forms the most conception. Remarkably, in communication interference is an inevitable price, as imaginary by technologist in an optimum communication theme. Random cryptography technique, from a few years is viewed as a creative speculative means that for the resilient of capability of channel. The event and therefore the renovation of pseudo-random codes, example turbo codes and Low-Density Parity Check (LDPC) codes [7-10], proves the real-world result of reliability values.

Forward Error Correction (FEC) or channel coding [11,12] is an exemplary method to expand the presentation and unwavering quality of a correspondence channel. At the point when re-transmission is 
moderately expensive and the framework is post pone delicate, FEC is utilized in the correspondence framework. When FEC is applied in a correspondence framework, controlled repetition is acquainted with the communicated bundle at the transmitter utilizing Error Control Codes (ECC) that permits identification and amendment of a specific measure of mistake at the beneficiary side. Channel encoding activity is the way toward including repetition at the transmitter where the activity changes over data pieces to codewords. The blunder can be distinguished and fixed at the recipient during the channel translating activity. Consequently, it wipes out the requirement for re-transmission. This is the key comfort of FEC which supports defer delicate correspondence frameworks. The points of interest gave by FEC come a few extra expenses [13-17]. The utilization of FEC in Under Water Sensor Network (UWSN) is supported based on a few variables. The utilization of FEC in coded correspondence framework can accomplish a specific dependability at essentially lower Signal to Noise Ratio (SNR) than an un coded framework. The data in SNR among un coded and coded frameworks is called coding gain and the estimation of coding gain relies upon 3 factors: The sort of ECC, code rate and unraveling calculation (i.e., the intricacy of translating calculation). The lower signal force transmission prerequisite at the transmitter (because of coding gain) which comes at the expense of additional vitality utilization because of channel encoding, channel interpreting and the transmission of excess pieces presented by FEC [18-20]. In this way, we have to think about the additional force utilization acquainted due with encoding, deciphering and transmission of additional pieces and the force sparing because of utilization of FEC. The utilization of FEC is possibly legitimized if additional force utilization because of encoding, deciphering and additional transmission, is lower than the force sparing because of coding gain, due to vitality obliged nature of UWSN hub. To make a reasonable correlation among un coded and coded framework with assortment of balance arranges, the SNR is estimated as the proportion of vitality per bit to the upsetting demand. When sending text data from one node to another node in underwater important performance parameter is bit error rate and power, In this paper the characteristic of BER and power for different modulation techniques, different coding and different interleavers are verified. For simulation purpose MATLAB 2015 is used. Desired BER for text is $10^{-5}$. We propose an hybrid approach to reduce the burst error in underwater communication. The simple channel code convolution is not able to improve BER at decoding output. For this reason, turbo code is introduced to enhance the performance. OFDM saves the Bandwidth. MIMO technique increases the data rate. IDMA reduces channel noise such as burst error and fading. We propose an efficient and hybrid technique that combines MIMO OFDM with IDMA scheme to mitigating the fading issue in underwater wireless communications.

\section{System configuration}

Binary phase shift keying (BPSK)

In communication basic modulation technique is BPSK. $\sqrt{A_{b}}$ and $-\sqrt{A_{b}}$ symbols are used to represent binary data 1 and 0 . Mathematically it is written as;

$$
\begin{aligned}
& K_{1}(t)=\sqrt{\frac{2 A_{b}}{\tau_{b}}} \cos \left(\Omega_{c} t+\Phi\right)(\Phi=0) \\
& K_{2}(t)=\sqrt{\frac{2 A_{b}}{\tau_{b}}} \cos \left(\Omega_{c} t+\Phi+\pi\right)=-K_{1}(t)
\end{aligned}
$$

duration of bit is $\tau_{b}$, energy of bit is denotes as $A_{b}$, angular incidence is denoted as $\Omega_{c}$ and $\Phi$ represents the phase angle. $R$ is the received data, it is written mathematically as;

$R(t)=K(t)+N$

where $N$ is the noise. Bit error rate probability is given as;

$$
\begin{aligned}
& S_{e}\left(\frac{\bar{e}}{K_{2}(t)}\right)=S_{e}\left(\frac{\bar{e}}{0}\right) \\
& =\frac{1}{\sqrt{\pi N}} e^{\left[-\frac{1}{N}\left(e-K_{21}\right)^{2}\right]} \\
& =\frac{1}{\sqrt{\pi N}} e^{\left\{\left[\frac{-\left[e-\left(-\sqrt{A_{b}}\right)\right]}{N}\right]\right\}^{2}}
\end{aligned}
$$


$=\frac{1}{\sqrt{\pi N}} e^{\left[-\frac{1}{N}\left(e+A_{b}\right)^{2}\right]}$

when 0 is transmitted receiver accepts as 1 is represented as;

$\int_{0}^{\infty} S_{e}\left(\frac{\bar{e}}{0}\right) d r=E_{n}(0)$

$E_{n}(0)=\int_{0}^{\infty} e^{\left[-\frac{1}{N}\left(e+A_{b}\right)^{2}\right]} d r$

$\frac{1}{\sqrt{N}}\left(r+A_{b}\right)=\mathrm{N}$

Above equation can be written as;

$S_{n}(0)=\frac{1}{\sqrt{\pi}} \int_{\frac{\sqrt{A_{b}}}{N}}^{\infty} e^{\left[-\frac{1}{N}(z)^{2}\right]} \mathrm{dz}$

Multiplying Eq. (11) by 2;

$S_{n}(0)=\frac{1}{2} \frac{2}{\sqrt{\pi}} \int_{\frac{\sqrt{A_{b}}}{N}}^{\infty} e^{\left[-(z)^{2}\right]} \mathrm{dz}$

$S_{n}(0)=\frac{1}{2} \operatorname{erfc}\left(\sqrt{\frac{A_{b}}{N}}\right)$

$S_{n}(0)=P\left(\sqrt{\frac{2 A_{b}}{N}}\right)$

similarly;

$S_{n}(1)=\frac{1}{2} \operatorname{erfc}\left(\sqrt{\frac{A_{b}}{N}}\right)$

The complete BER for BPSK is represented as;

$\mathrm{BER}=\frac{1}{2} S_{n}(0)+\frac{1}{2} S_{n}$

In case of BPSK demodulation carrier frequency of sender and detector coherent signal should be equal. It is less complex compared to other modulation technique.

Quadrature phase shift keying (QPSK)

In QPSK 2 bits are used to represent symbol. QPSK is represented mathematically as [10];

$K_{1}(t)=\sqrt{\frac{2 A_{b}}{\tau_{s}}} \cos \left(2 \pi \Omega_{c} t+(2 j-1) \frac{\pi}{4}\right)$

It can be represented as;

$K_{1}(t)=\sqrt{\frac{2 A_{b}}{\tau_{s}}} \cos \left((2 j-1) \frac{\pi}{4}\right) \operatorname{cosine} \Omega_{c} t-\sqrt{\frac{2 A_{b}}{\tau_{s}}} \operatorname{sine}\left((2 j-1) \frac{\pi}{4}\right) \operatorname{sine} \Omega_{c} t$

here $\mathrm{j}=1,2,3,4 . \tau_{s}$ are duration of symbol. The final output is;

$e_{1}(t)=K_{1}(t)+N$

$\mathrm{N}$ is the noise. 
In $K_{4}(t)$ the correct data is sent as;

$\left.E_{K_{4}(t)}=\int_{0}^{\infty} \frac{1}{\sqrt{\pi N}} e^{\left[\left[-\frac{1}{N}\left(e_{1}-\sqrt{\frac{A_{b}}{2}}\right)^{2}\right]\right]} d r_{1} \int_{0}^{\infty} \frac{1}{\sqrt{\pi N}} e^{\left[\left[-\frac{1}{N}\left(e_{1}-\sqrt{\frac{A_{b}}{2}}\right)^{2}\right]\right.}\right] d r_{2}$

$e_{1}$ and $e_{2}$ are independent, $\frac{e_{i}-\sqrt{\frac{A_{b}}{2}}}{\sqrt{N}}$ is substituted as $\mathrm{r}$. Here $\mathrm{i}=1,2$.

$E_{K_{4}(t)}=\left[\frac{1}{\sqrt{\pi}} \int_{-\sqrt{\frac{E_{b}}{N}}}^{\infty} e^{\left[-(z)^{2}\right]}\right]^{2} \mathrm{dz}$

That is;

$\frac{1}{\sqrt{\pi}} \int_{-a}^{\infty} e^{\left[-x^{2}\right]} \mathrm{dx}=1-\frac{1}{2} \operatorname{erfc}(c)$

Can be written as;

$E_{K_{4(t)}}=\left[1-\frac{1}{2} \operatorname{erfc}\left(\sqrt{\frac{A_{b}}{2 N}}\right)\right]^{2}$

Error is denoted by;

$\xi_{e K 4(t)}=1-\xi_{K 4(t)}$

$\xi_{e}=\frac{1}{2} \operatorname{erfc}\left(\sqrt{\frac{A_{b}}{N}}\right)$

In QPSK communication system 1 symbol is represented by 2 binary data. That consumes the more power. In underwater communication QPSK technique is very complicated.

Quadrature amplitude modulation (QAM)

In QAM phase and magnitude variation is used to characterize bits, both amplitude and phase QAM is represented as;

$K_{i}(t)=\sum_{l=-\infty}^{\infty} \sqrt{\frac{A \tau_{Q}}{\tau}} h_{\tau}(t-l \tau) \operatorname{cosine}\left(2 J_{l}+1-\sqrt{L}\right)$

$K_{Q}(t)=\sum_{l=-\infty}^{\infty} \sqrt{\frac{A \tau_{Q}}{\tau}} h_{\tau}(t-l \tau) \operatorname{sine}\left(2 J_{l}+1-\sqrt{L}\right)$

$A \tau_{Q}$ is energy of symbol, where $L=4$.

BER is considered as;

$E_{O, M Q A M}=\frac{1}{\log _{2} \sqrt{M}}\left(1+2+4 \ldots \ldots .+2^{\log _{2} \sqrt{M-1}}\right) E_{p}$

in general;

$E_{O, M Q A M}=\frac{4}{\log _{2} L}\left(1-\frac{1}{\sqrt{L}}\right) \sum_{i=1}^{\sqrt{\frac{L}{2}}} P\left((2 j-1) \sqrt{\frac{3 A_{b} \log _{2} L}{(L-1) N}}\right)$ 
underwater communication is limited by battery power as solar energy cannot be used to charge the battery. As QAM receiver circuit is extra complex when compared to other modulation techniques.

\section{Materials and methods}

In this paper, we assess the consumed power of convolutional code, turbo code. The code which consumes less power is recognized, assessment is based on the hypothesis that error control performance that is assessed by bit error rate examination. In this paper user-specific interleavers (random and matrix interleaving) are used to completely differentiate different users. For planet suitableness these interleavers is selected haphazardly and it's not needed that they're orthogonal. In communication system interference is unpredictable between the users, for multiple access channels at first Interleaver Division Multiple Access (IDMA) was planned, same construct is being applied for alternative requests, like transmission schemes, coded tone, antenna systems. IDMA theme offers the flexibility and power. At the supply finish, mistreatment forward error correction encoder information is initially encoded for sender $b_{i}$ with rate as $\mathrm{t}$ and therefore the encoded data is interleaved by an interleaver into a chip order. Before sending the information, modulation is employed as in Figure 1. The users are unit separated by specific interleavers, and it is not needed to use predictable spreading method employed in CDMA. Multiple Input Multiple Output Orthogonal Frequency Division Multiplexing Interleaver Division Multiple Access (MIMO OFDM IDMA) is represented (Figure 1). In this process we assume that in a single communication cell $k$ users are arbitrarily spread. The following assumption is made: $T t$ transmitting antennas are used at the transmitter and $\operatorname{Tr}$ receiving antennas are used at the receiver.

Let $b_{i}$ denotes the input bit stream representing to the $i^{\text {th }}$ user. Using turbo code/ convolution coding, we first encode the bit stream. Them using random / matrix interleaving encoded data is inreleaved. Binary phase shift keying (BPSK) modulation method is used on encoded and interleaving bit stream. Here $m_{i}$ represents the bit stream to be transmitted to the $\mathrm{i}^{\text {th }}$ user after performing channel coding.

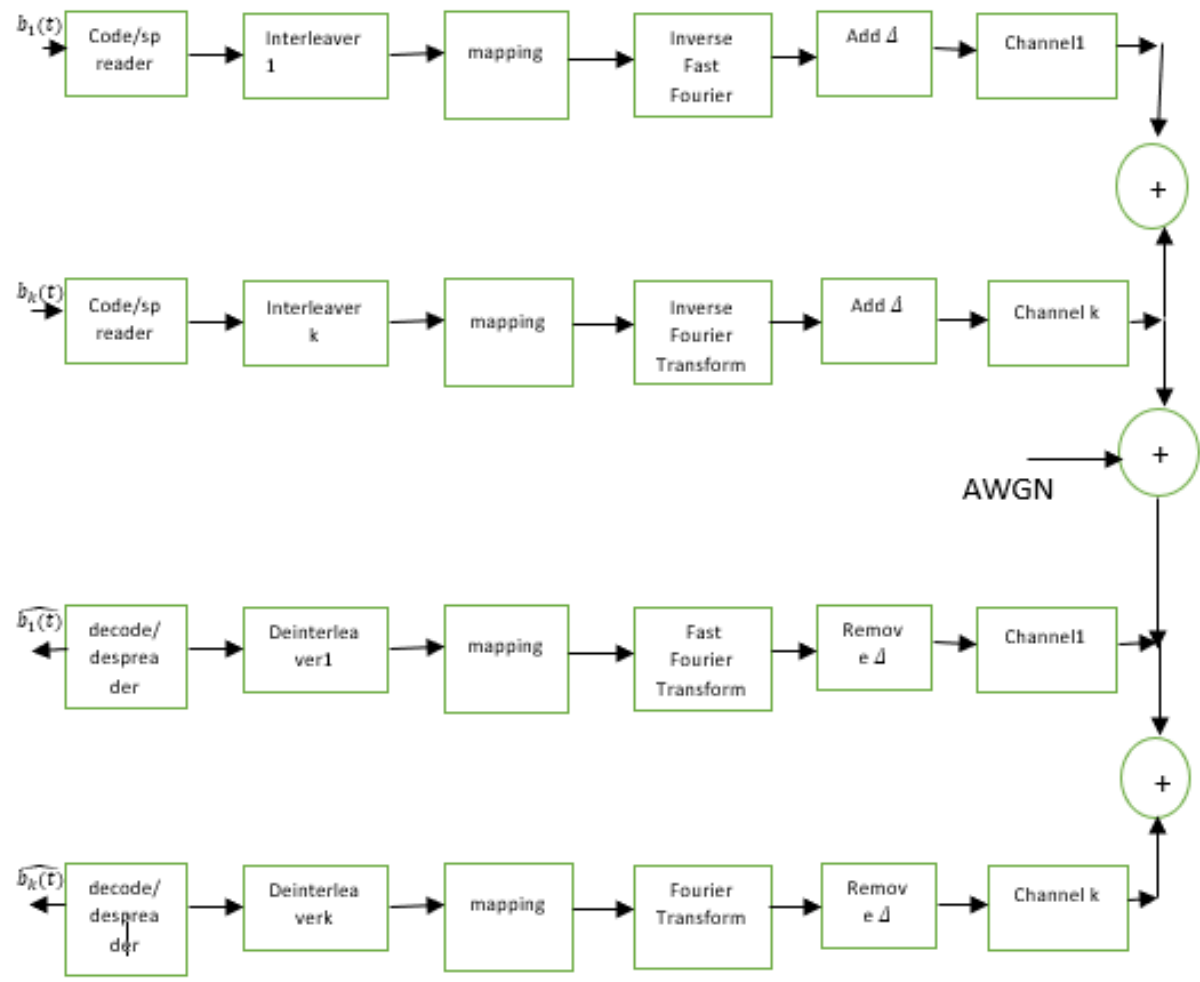

Figure 1 MIMO OFDM IDMA. 


\section{Results and discussion}

Convolutional coding technique with random interleaving and 3 modulation techniques

Figure 2(a) compares the consumed power and in Figure 2(b) compares the bit error rate of MIMO OFDM IDMA for UWAN. In Figure 2 for coding we take convolution code, for interleaving we take random interleaver and 3 modulation techniques BPSK, QPSK, QAM. Simulation results shows that consumed power for BPSK is $31 \mathrm{~dB}$ and for QPSK and QAM is around $35 \mathrm{~dB}$ for $\mathrm{E}_{\mathrm{b}} / \mathrm{N}_{\mathrm{o}} 12$ to $14 \mathrm{~dB}$ and bit error rate is around $10^{-3}$ for SNR 13 to $15 \mathrm{~dB}$.

(a)

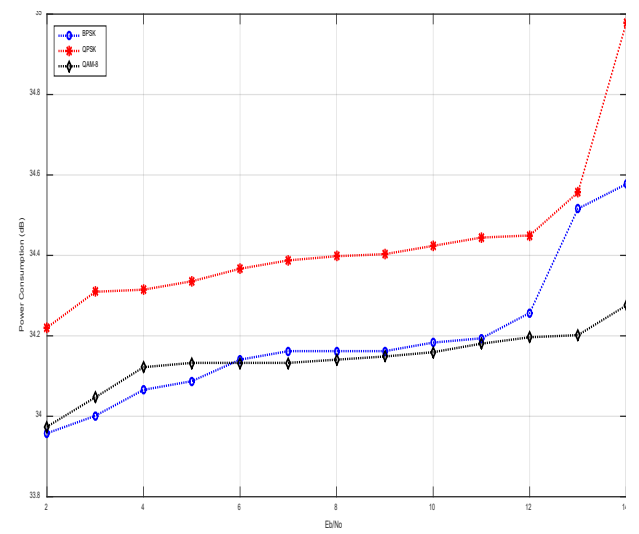

(b)

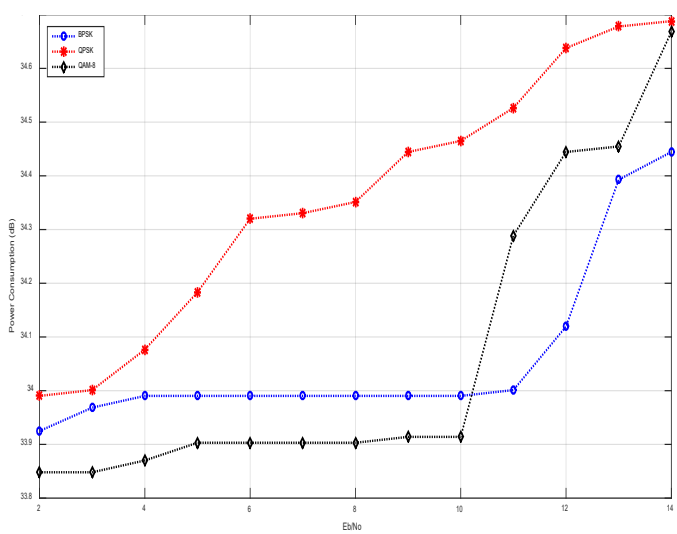

Figure 2 (a) Power consumption, and (b) BER for convolution coding and random interleaving method.

\section{Convolutional coding technique with matrix interleaving and 3 modulation techniques}

Figure 3(a) compares consumed power and in Figure 3(b) compares the bit error rate of MIMO OFDM IDMA for UWAN. In Figure 3 for coding we take Convolution code, for interleaving we take Matrix interleaver and 3 modulation techniques: BPSK, QPSK, QAM. Simulation results shows that consumed power for BPSK is $31 \mathrm{~dB}$ and for QPSK and QAM is around $35 \mathrm{~dB}$ for $\mathrm{E}_{\mathrm{b}} / \mathrm{N}_{\mathrm{o}} 12$ to $14 \mathrm{~dB}$ and bit error rate is around $10^{-3}$ for SNR 13 to $15 \mathrm{~dB}$.

(a)

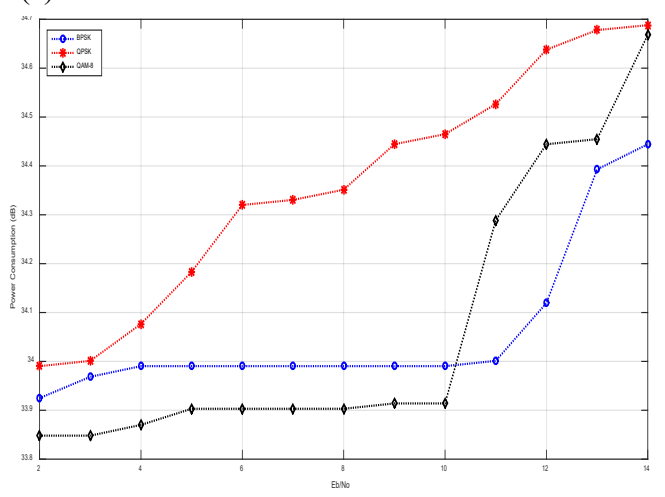

(b)

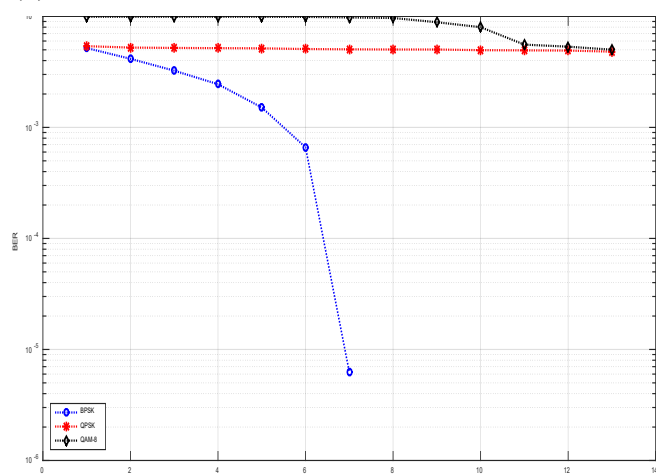

Figure 3 (a) Power consumption and (b) Bit error rate for convolution coding and matrix interleaving method.

Turbo coding technique with random interleaving and 3 modulation techniques

Turbo code is a new class of Convolutional code. Turbo code gives higher performance when compared to convolution code. Figure 4(a) compares consumed power and in Figure 4(b) compares the bit error rate of MIMO OFDM IDMA for UWAN. In Figure $\mathbf{4}$ for coding we take turbo code, for 
interleaving we take random interleaver and 3 modulation techniques BPSK, QPSK, QAM. Simulation results shows that consumed power is around $34 \mathrm{~dB}$ for $\mathrm{E}_{\mathrm{b}} / \mathrm{N}_{\mathrm{o}} 12$ to $14 \mathrm{~dB}$ and bit error rate for BPSK is $10^{-6}$ and for QPSK and QAM is around $10^{-3}$ for SNR 13 to $15 \mathrm{~dB}$.

(a)

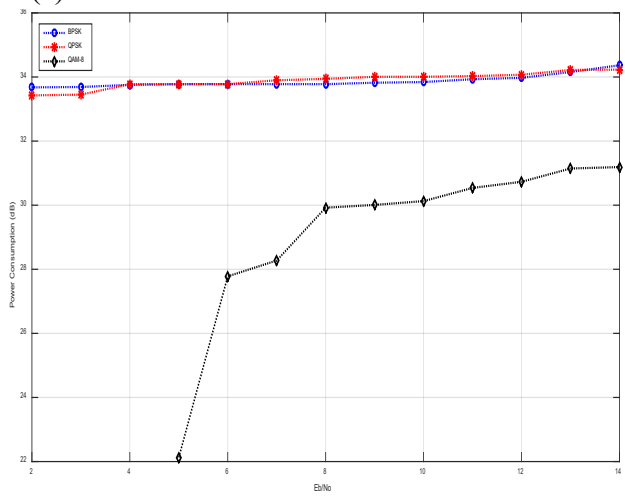

(b)

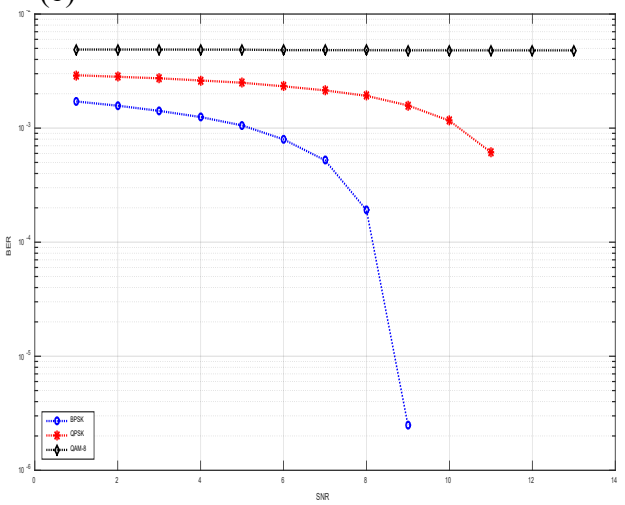

Figure 4 (a) Power consumption and (b) Bit error rate for turbo coding and random interleaving method.

\section{Turbo coding with matrix interleaving}

Figure 5(a) compares consumed power and in Figure 5(b) compares the bit error rate of MIMO OFDM IDMA for UWAN. In Figure 5 for coding we take turbo code, for interleaving we take matrix interleaver and 3 modulation techniques BPSK, QPSK, QAM. Simulation results shows that consumed power for BPSK and QPSK is $34 \mathrm{~dB}$ and consumed power for QAM is $29 \mathrm{~dB}$ for $\mathrm{E}_{\mathrm{b}} / \mathrm{N}_{\mathrm{o}} 12$ to $14 \mathrm{~dB}$ and bit error rate for BPSK, QPSK and QAM is $10^{-3}$ for SNR 13 to $15 \mathrm{~dB}$.

(a)

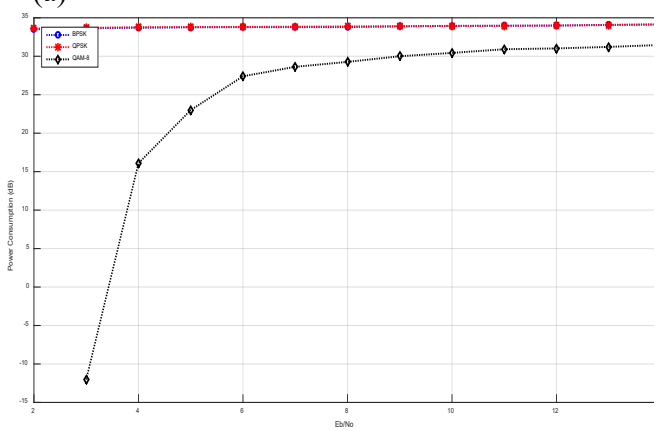

(b)

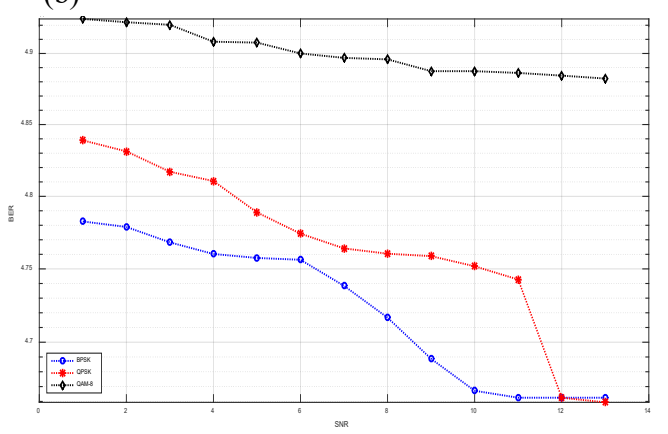

Figure 5 (a) Power consumption and (b) Bit error rate for turbo coding and matrix interleaving method

\section{Performance parameters comparison}

For error correction turbo codes are very powerful tool when combined with interleaver gives better results. Performance parameters are compared for Orthogonal Frequency Division Multiplexing Interleaver Division Multiple Access Multiple Input Multiple Output by captivating 2 coding methods convolutional code and turbo code, with 2 interleaving method random interleaver, matrix interleaver and 3 modulation methods BPSK, QAM and QPSK. Results are tabulated in Table 1. We observe from simulation results that turbo code with binary phase shift keying and random interleaving improves the bit error rate performance. 
Table 1 Evaluation of performance constraints.

\begin{tabular}{ccccc}
\hline $\begin{array}{c}\text { Modulation } \\
\text { method }\end{array}$ & Coding method & $\begin{array}{c}\text { Interleaving } \\
\text { technique }\end{array}$ & $\begin{array}{c}\text { Bit error } \\
\text { rate }\end{array}$ & $\begin{array}{c}\text { Consumption of } \\
\text { power }\end{array}$ \\
\hline BPSK & Convolutional code & Random interleaving & $10^{-3}$ & $31 \mathrm{~dB}$ \\
\hline QPSK & Convolutional code & Random interleaving & $10^{-3}$ & $35 \mathrm{~dB}$ \\
\hline QAM & Convolutional code & Random interleaving & $10^{-3}$ & $35 \mathrm{~dB}$ \\
\hline BPSK & Convolutional code & Matrix interleaving & $10^{-3}$ & $31 \mathrm{~dB}$ \\
\hline QPSK & Convolutional code & Matrix interleaving & $10^{-3}$ & $35 \mathrm{~dB}$ \\
\hline QAM & Convolutional code & Matrix interleaving & $10^{-3}$ & $35 \mathrm{~dB}$ \\
\hline BPSK & Turbo code & Random interleaving & $10^{-6}$ & $36 \mathrm{~dB}$ \\
\hline QPSK & Turbo code & Random interleaving & $10^{-3}$ & $35 \mathrm{~dB}$ \\
\hline QAM & Turbo code & Random interleaving & $10^{-3}$ & $33 \mathrm{~dB}$ \\
\hline BPSK & Turbo code & Matrix interleaving & $10^{-3}$ & $36 \mathrm{~dB}$ \\
\hline QPSK & Turbo code & Matrix interleaving & $10^{-3}$ & $36 \mathrm{~dB}$ \\
\hline QAM & Turbo code & Matrix interleaving & $10^{-3}$ & $32 \mathrm{~dB}$ \\
\hline
\end{tabular}

\section{Conclusions}

Due to limited energy and reliability, underwater acoustic wireless communication is postured to numerous issues and challenges. Sending information in underwater wireless communication using acoustic link is challenging. In this paper we present reliable channel coding with MIMO OFDM IDMA which provides reliability. This technique provides reliable communication from source to destination. In underwater node energy is very important parameter. From Simulation result we conclude that convolution coding with random and matrix Interleaving gives power up to $30 \mathrm{~dB}$ and BER $10^{-3}$. The performance is improved when we combine turbo code with random interleaving and BPSK gives BER up to $10^{-6}$ and power $36 \mathrm{~dB}$. Simulation result offers the transaction between BER and consumed power. As a future work the physical layer is combined with network layer as a cross layer approach to improve the performance.

\section{References}

[1] H Luo, K Wu, R Ruby, F Hong, Z Guo and LM Ni. Simulation and experimentation platforms for underwater acoustic sensor networks: Advancements and challenges. ACM Comput. Surv. 2017; 50, $1-44$.

[2] K Pelekanakis and M Chitre. New sparse adaptive algorithms based on the natural gradient and the $\mathrm{L}_{0}$-norm. IEEE J. Ocean. Eng. 2013; 38, 323-33.

[3] YV Zakharov and AK Morozov. OFDM transmission without guard interval in fast-varying underwater acoustic channels. IEEE J. Ocean. Eng. 2015; 40, 144-58.

[4] Z Wang, S Zhou, J Catipovic and P Willett. Asynchronous multiuser reception for OFDM in underwater acoustic communications. IEEE Trans. Wireless Comm. 2013; 12, 1050-61.

[5] SS Shahapur and R Khanai. Underwater sensor network at physical, data link and network - layer a survey. In: Proceedings of the 2015 International Conference on Communications and Signal Processing, Melmaruvathur, India. 2015.

[6] Y Chen, ZH Wang, L Wan, H Zhou, S Zhou and X Xu. OFDM modulated dynamic coded cooperation in underwater acoustic channels. IEEE J. Ocean. Eng. 2015; 40, 159-68.

[7] R Khanai, SS Shahapur and DA Torse. Performance analysis of underwater acoustic communication using IDMA-OFDM-MIMO with reed solomon and turbo code. Int. J. Comput. Network Inform. Sci. 2018; 12, 41-6.

[8] Z Chen, J Wang and YR Zheng. Frequency-domain turbo equalization with iterative channel estimation for MIMO underwater acoustic communications. IEEE J. Ocean Eng. 2017; 42, 711-21.

[9] KK Redo, P Djukicand and P Mohapatra. STUMP: Exploiting position diversity in the staggered TDMA underwater MAC protocol. In: Proceedings of the IEEE INFOCOM 2009. Rio de Janeiro, Brazil. 2009, p. 2961-5. 
[10] J Ma and W Lou. Interference-aware spatio-temporal link scheduling for long delay underwater sensor networks. In: Proceedings of the $8^{\text {th }}$ Annual IEEE Communications Society Conference on Sensor, Mesh and Ad Hoc Communications and Networks, Salt Lake City, UT, 2011, 431-9.

[11] M Ayaz, I Baig, A Abdullah and I Faye. A survey on routing techniques in underwater wireless sensor networks. J. Network Comput. Appl. 2011; 34. 1908-27.

[12] JP Jornet, M Stojanovic and M Zorz. Focused beam routing protocol for underwater acoustic networks. In: Proceedings of the $3^{\text {rd }}$ ACM International Workshop on Underwater Network, San Francisco. 2008, p.75-82.

[13] J Chen, X Wu and G Chen. REBAR: A Reliable and energy balanced routing Algorithm for UWSNs. In: Proceedings of the $7^{\text {th }}$ International Conference on Grid and Cooperative Computing, Shenzhen, China. 2008, p. 349-55.

[14] Z Chen, J Wang and YR Zheng. Frequency-domain turbo equalization with iterative channel estimation for MIMO underwater acoustic communications. IEEE J. Ocean Eng. 2017; 42, 711-21.

[15] Z Wang, S Zhou, J Catipovic and P Willett. Asynchronous multiuser reception for OFDM in underwater acoustic communications. IEEE Trans. Wireless Comm. 2013; 12, 1050-61.

[16] H Luo, K Wu, R Ruby and M Ni Lionel. Simulation and experimentation platforms for underwater acoustic sensor networks: Advancements and challenges. ACM Comput. Surv. 2017; 50, 28.

[17] D Shin, D Hwang and D Kim. DFR: An efficient directional flooding-based routing protocol in underwater sensor networks. Wireless Comm. Mobile. Comput. 2012; 12, 1517-27.

[18] M Hill, P Wang, Y Noh, L Vieira, M Gerla and JH Cui. Pressure routing for underwater sensor networks. In: Proceedings of the 2010 IEEE INFOCOM, San Diego, CA, USA. 2020.

[19] H Yan, J Shi and JH Cui. DBR: Depth-based routing for underwater sensor networks. In: Proceedings of the Networking, Singapore, Singapore. 2008, p.72-86.

[20] D Shin, D Hwang and D Kim. DFR: An efficient directional flooding-based routing protocol in underwater sensor networks. Wireless Comm. Mobile Comput. 2012; 12, 1517-27.

[21] L Hu and D Evans. Localization for mobile sensor networks. In: Proceedings of the $10^{\text {th }}$ Annual International Conference on Mobile Computing and Networking, Philadelphia, Pennsylvania. 2004, p. $45-57$.

[22] Z Zhou, J Cui and S Zhou. Localization for large-scale underwater sensor networks. Technical report, UCONN CSE Rep. UbiNet-TR06-04. 2006.

[23] YV Zakharov and AK Morozov. OFDM transmission without guard interval in fast-varying underwater acoustic channels. IEEE J. Ocean. Eng. 2015; 40, 144-58.

[24] N Li, JF Martinez, M Chaus and M Eckert. A survey on underwater acoustic sensor network routing protocols. Sensors 2016; 16, 414.

[25] F Rosas, RD Souz, ME Pellenz, C Oberli, G Brante, M Verhelst and S Pollin. Optimizing the code rate of energy-constrained wireless communications with HARQ. IEEE Trans. Wireless Comm. 2016; 15, 191-205.

[26] R Khanai, GH Kulkarni and DA Torse. Neural crypto-coding as DES: Turbo over land mobile satellite (LMS) channel. In: Proceedings of the 2014 International Conference on Communication and Signal Processing, Melmaruvathur, India. 2014.

[27] M Liang, J Duan, D Zhao, J Si and X Song. Novel joint encoding/decoding algorithms of fountain codes for underwater acoustic communication. J. Syst. Eng. Electron. 2016; 27, 772- 9.

[28] R Coates. Underwater acoustic systems. Wiley, New York, 1989.

[29] J Huang, JZ Huangand and CR Berger. Iterative sparce channel estimation and decoding for underwater MIMO-OFDM. EURASIP J. Adv. Signal Process. 2010; 2010, 460379.

[30] G Barreto, DSimao, ME Pellenz, RD Souza, EJamhour, MC Penna, G Brante and BS Chang. Energy-efficient channel coding strategy for underwater acoustic networks. Sensors 2017; 17, 728.

[31] S More and KK Naik. Modelling and simulation of IDMA-OFDM for underwater acoustic communication. Defence Sci. J. 2015; 65, 307-11. 